\title{
Impairment Modeling for Joint Transmission CoMP
}

\author{
Konstantinos Manolakis ${ }^{1}$, Lars Thiele ${ }^{1}$, Christian Oberli ${ }^{2}$, Thomas Haustein $^{1}$, Volker Jungnickel ${ }^{1}$ \\ ${ }^{1}$ Fraunhofer Institute for Telecommunications, Heinrich Hertz Institute, Einsteinufer 37, D-10587 Berlin, Germany \\ ${ }^{2}$ Pontificia Universitad Católica de Chile, Vicuña Mackenna 4860, Santiago, Chile \\ Email: Konstantinos.Manolakis@hhi.fraunhofer.de
}

\begin{abstract}
Base station coordination is envisioned as a key technology in future mobile networks. It reduces the interference between adjacent cells and promises enhancement of spectral efficiency. However, little is known about the penalties due to impairments. Channel errors may be caused by channel estimation, feedback delay as well as by synchronization errors among the base stations. Hence, the computation of the precoder relies on imprecise information and the orthogonality between the user data is disturbed. In this paper, we give an overview on potential impairment effects. We derive an accurate signal model capturing the effect of synchronization impairments depending on the feedback delay. The resulting interference levels must not be larger than the one obtained after cooperation of the base stations in the cluster being limited by interference of noncoordinated base stations surrounding the cluster. We introduce an equivalent mean square error for these impairment effects, propose a practical way to evaluate the corresponding performance degradation and discuss compensation mechanisms.
\end{abstract}

\section{INTRODUCTION}

Multiple antenna techniques denoted as Multiple-InputMultiple-Output (MIMO) promise a significant increase of the spectral efficiency in mobile networks compared to traditional single-antenna techniques, in particular if they are used to serve multiple users on the same radio resource [1], [2]. More practical methods separate the users signals by zero-forcing (ZF) precoding [3], [4]. However, in multi-cell networks, there is substantial interference between the cells limiting the overall performance.

Using joint transmission (JT) coordinated multi-point (CoMP) in mobile networks, the idea of MIMO is generalized. The antennas of multiple distributed base stations are considered as inputs and the antennas of the multiple terminals in these cells are regarded as outputs of a distributed MIMO system. By synchronizing the base stations and enabling data and channel state information exchange [5], the mobile network is enabled to transmit jointly to all terminals so that the interference between the cells is reduced. This technology has received considerable interest in recent research. Early field trials based on the Third Generation Partnership Project (3GPP) Long Term Evolution (LTE) have been conducted in several scenarios [6].

During these trials, the sensitivity of JT CoMP against impairments became obvious. There is little known in the literature about the performance degradation due to imperfect channel knowledge and synchronization errors. Channel errors can be caused by channel estimation, feedback quantization, feedback delay as well as by time and frequency synchronization misalignments among the oscillators of the cooperative base stations. In [7] the estimation of multiple frequency offsets is investigated. The required frequency accuracy has been studied in [8]. In [9], the performance degradation due to imperfect synchronization and channel knowledge is studied in an Orthogonal Frequency Division Multiplexing (OFDM)based framework while applying JT CoMP. More recent work [10] has extended the general mathematical framework and evaluated distributed base stations with individual synchronization errors.

Our objective in this paper is to provide an overview on the impairment effects in a cellular network using JT CoMP and propose a practical way to model and evaluate the corresponding performance degradation. We provide early results for the impact of synchronization impairments in isolated clusters and argue that the related interference must be less than the residual interference due to non-coordinated cells surrounding the cluster in a real network where JT CoMP is performed.

The paper is organized as follows. In section II, the channel and synchronization impairments are quantified using a unified system model for JT CoMP. In section III, the performance degradation due to synchronization errors is analyzed and evaluated. Section IV discusses the effect of clustering on the achievable throughput. We show how the performance is reduced by impairments. Finally we draw our conclusions and discuss open issues in section VI.

\section{Signal Model for JT COMP With CHANNEL AND SYNCHRONIZATION IMPAIRMENTS}

In general full knowledge of the channel matrix is required for the JT CoMP downlink at the transmitter's side. In the most simple case (ZF), linear precoding is realized by multiplication of the transmit signals with the pseudo-inverse of the channel matrix, i.e. $\mathbf{P}=\mathbf{H}^{-1}{ }^{1}$. We assume $\vec{s}$ to be a $K \times 1$ data vector where one stream is transmitted to each of $K$ single-antenna terminals and $\vec{y}=\mathbf{H} \vec{x}+\vec{w}$ the downlink MIMO system equation. Vector $\vec{x}$ is jointly transmitted from all base stations and $\vec{w}$ is an additive Gaussian noise (AWGN) vector. We apply $\vec{x}=\mathbf{P} \vec{s}$ so that the system equation reads $\vec{y}=\mathbf{H P} \vec{s}+\vec{w}=\vec{s}+\vec{w}$, which equals the transmitted signal plus noise.

However, we have to provide $\mathbf{H}$ to the base stations. In frequency division duplexing systems downlink and uplink are operated at different frequencies. Hence, the downlink channel is estimated by the mobile terminals and fed back to the base stations via an uplink signaling channel.

\footnotetext{
${ }^{1}$ The notation ${ }^{-1}$ is used to refer to the Moore-Penrose pseudo-inverse.
} 


\section{A. Channel Aging}

In practical systems there is always a delay between the channel estimation at the mobile terminal and the time instant when this estimate is used for composing the following downlink transmission. As the channel may change during this delay time, the channel observation may be outdated for precoding the next transmission. Reference [11] provides a characterization of the channel evolution due to this aging process for point-to-point MIMO links. However it assumes equal channel gain for all the links, which is not appropriate for JT CoMP.

In order to describe the effect of outdated channel knowledge, we extend the above system model description. We define $\vec{s}(n)$ as the data symbol vector to be transmitted on the downlink and $\mathbf{H}(n)$ as the MIMO channel matrix at time $n$. $\mathbf{P}(n, \tau)$ denotes the precoding matrix used at time $n$ but based on a $\tau$-old estimate of the channel, i.e., $\mathbf{P}(n, \tau)=\mathbf{H}^{-1}(n-\tau)$. We may also define a difference matrix $\mathbf{V}(n, \tau)$ that relates the $\tau$-old channel inverse with the current channel inverse as $\mathbf{H}^{-1}(n)=\mathbf{H}^{-1}(n-\tau)+\mathbf{V}(n, \tau)$. Then,

$$
\begin{aligned}
\vec{y}(n) & =\mathbf{H}(n) \mathbf{P}(n, \tau) \vec{s}(n)+\vec{w}(n) \\
& =\mathbf{H}(n) \mathbf{H}^{-1}(n-\tau) \vec{s}(n)+\vec{w}(n) \\
& =\mathbf{H}(n)\left[\mathbf{H}^{-1}(n)-\mathbf{V}(n, \tau)\right] \vec{s}(n)+\vec{w}(n) \\
& =\vec{s}(n)-\mathbf{H}(n) \mathbf{V}(n, \tau) \vec{s}(n)+\vec{w}(n) \\
& =\vec{s}(n)-\mathbf{V}^{\prime}(n, \tau) \vec{s}(n)+\vec{w}(n) \neq \vec{s}(n) .
\end{aligned}
$$

From Eq. (1) it becomes clear that the feedback delay $\tau$ causes inter-user interference depending on the matrix $\mathbf{V}^{\prime}$, as it breaks the inverse relationship between the channel and the precoder.

\section{B. Channel Estimation and Compression}

The precoding matrix $\mathbf{P}(n)$ is computed in practice from estimates of the channel matrix rather than from ideal knowledge of it. Thus, the matrix $\mathbf{V}(n)$ in Eq. (1) could be regarded as the precoding error due to channel estimation, which results in inter-user interference. Furthermore, as the signaling channel demands physical resources, solutions that rely on partial channel state information (CSI) at the transmitter have been proposed for practical implementation. These techniques are known as "limited feedback schemes". Compression and quantization of CSI imposes channel errors at the transmitter side, which can also be described by difference matrix $\mathbf{V}^{\prime}(n)$.

\section{Synchronization Errors}

It is well known that OFDM systems are sensitive to impairments as carrier frequency offset (CFO) and sampling frequency offset (SFO). For JT CoMP, synchronization conditions are substantially more challenging than for transmissions from a single base station, as the distributed base stations are driven by their local oscillators. This leads to individual CFOs and SFOs between the base stations as well as between each base station and each terminal. In our system model we consider a matrix $\boldsymbol{\Phi}(n)$ that contains the phase drifts caused by CFO and SFO between all bases and all terminals at time $n$. Then, the MIMO downlink transmission is described by

$$
\vec{y}(n)=\mathbf{H}(n) \boldsymbol{\Phi}(n) \mathbf{P}(n, \tau) \vec{s}(n)+\vec{w}(n) .
$$

If the phase drifts in $\boldsymbol{\Phi}(n)$ are small enough, the small angle approximation $e^{j \theta} \approx 1+j \theta$ can be applied to each element of $\boldsymbol{\Phi}(n)$. We re-write $\boldsymbol{\Phi}(n)$ as $\boldsymbol{\Phi}(n) \approx \mathbf{I}+j \boldsymbol{\Theta}(n)$. Then, Eq. (2) can be written again in the form

$$
\vec{y}(n)=\vec{s}(n)-\mathbf{H}(n) \mathbf{V}(n) \vec{s}(n)+\vec{w}(n) \neq \vec{s}(n)
$$

from which it becomes clear that $\mathbf{V}(n)=j \boldsymbol{\Theta}(n) \mathbf{P}(n)$ and that $\boldsymbol{\Theta}(n) \neq \mathbf{0}$ breaks the inverse relationship between channel $\mathbf{H}(n)$ and precoder $\mathbf{P}(n)$.

\section{Relation Between $\mathbf{V}^{\prime}$, Equivalent Channel MSE and SIR}

From above analysis, it is obvious that the signal model for all these impairments has the same algebraic structure. The error matrix $\mathbf{V}$ quantifies the mismatch between the applied precoder $\mathbf{P}$ and the actual channel $\mathbf{H}$. As a consequence, mobile users suffer from inter-user interference. If we don't considering any error in the precoder but instead we consider a general channel error $\Delta \mathbf{H}$ we have

$$
\vec{y}=(\mathbf{H}+\boldsymbol{\Delta} \mathbf{H}) \mathbf{P} \vec{s}+\vec{w}
$$

and obtain the relation

$$
\vec{y}=\vec{s}+\Delta \mathbf{H P} \vec{s}+\vec{w} .
$$

In case of zero-forcing, $\mathbf{P}=\mathbf{H}^{-1}$ and by comparing with Eq. (1), $\mathbf{V}^{\prime}=\Delta \mathbf{H H}^{-1}$. For intuition, if $\mathbf{V}^{\prime}, \boldsymbol{\Delta} \mathbf{H}$ and $\mathbf{H}$ would be scalars then $V^{\prime}=\frac{\Delta H}{H}$. Hence, we can treat any of the above impairments as if they would cause an equivalent channel estimation error. We may quantify the expectation of this mean square error (MSE) depending on system parameters such as the feedback delay, the granularity of the channel quantization or the synchronization errors. Next we determine the system performance in terms of SIR equivalent to the expectation of MSE:

$$
S I R=\frac{\mathcal{E}\left(|s|^{2}\right)}{\mathcal{E}\left(\left|V^{\prime} s\right|^{2}\right)}=\frac{\mathcal{E}\left(|H|^{2}\right)}{\mathcal{E}\left(|\Delta H|^{2}\right)}=\frac{1}{M S E} .
$$

In this way, we can specify the maximum tolerable channel MSE for the system and therefrom the related physical parameters of the impairments.

\section{IMPACT OF SYNCHRONIZATION IMPAIRMENTS}

\section{A. SIR analysis}

In this section we take a closer look at time and frequency synchronization misalignments at the base stations, analyze this error source and evaluate its impact on the JT CoMP performance degradation. We have studied and mathematically modeled imperfect time and frequency synchronization in an OFDM-based MIMO system in [10], derived signal-tointerference ratio (SIR) levels and verified by simulations.

The downlink spectrum observed on a random subcarrier $k$ at any given receive branch $j$ is

$$
y_{j}(k)=u_{j}(k)+\bar{u}_{j}(k)+w_{j}(k),
$$


where $u_{j}(k)$ represents the frequency spectrum of the received multi-user signal at receive antenna $j$ for transmitted subcarrier $k$ and $\bar{u}_{j}(k)$ is the received spectrum of the multi-user signal of all other subcarriers $\nu \neq k$. All mobiles are equipped with a single antenna and ZF precoding is applied on each subcarrier by assuming perfect channel knowledge. The on-carrier signal is given by $u_{j}(k)=s_{j}(k)+\bar{s}_{j}(k)$, where $s_{j}(k)$ is the desired signal of user $j$ and $\bar{s}_{j}(k)$ is the interference observed by user $j$ due to the loss of inter-user orthogonality of the precoded transmission due to synchronization misalignments given by

$$
\bar{s}_{j}(k)=\jmath \sum_{u=1}^{N_{R}} s_{u}(k) \sum_{i=1}^{N_{T}} \theta_{j i}(k) p_{i u}(k) H_{j i}(k),
$$

where $N_{T}$ and $N_{R}$ denote the total number of transmit and receive branches respectively. The inter-carrier signal in Eq. (7) is equal to

$$
\bar{u}_{j}(k)=\sum_{\substack{\nu=-\frac{N_{s}}{2} \\ \nu \neq k}}^{\frac{N_{s}}{2}-1} \sum_{u=1}^{N_{R}} s_{u}(\nu) \sum_{i=1}^{N_{T}} \phi_{j i}(k, \nu) p_{i u}(\nu) H_{j i}(\nu)
$$

and expresses the inter-carrier interference (ICI) from $N_{s}-1$ subcarriers that affects subcarrier $k$ of user $j$. Quantities $\theta_{j i}, \phi_{j i}$ and $p_{i j}$ are complex valued entries of the phase drift matrices $\boldsymbol{\Theta}(k), \boldsymbol{\Phi}(k)$ and of the precoding matrix $\mathbf{P}(k)$ respectivly. The CFO and SFO effect is captured by $\boldsymbol{\Theta}(k)$ and $\boldsymbol{\Phi}(k)$; exact mathematical expressions are given in [10].

Finally a "signal to on-carrier plus inter-carrier interference ratio" of a downlink signal received by mobile $j$ on subcarrier $k$ is defined as

$$
\operatorname{SIR}_{j}(k)=\frac{\left|s_{j}(k)\right|^{2}}{\left|\bar{s}_{j}(k)+\bar{u}_{j}(k)\right|^{2}} .
$$

\section{B. Numerical evaluation}

For evaluating of the mobile's SIR, or the equivalent channel MSE caused by synchronization errors, we use typical 3GPP LTE system parameters [12]. We consider $N_{s}=2048$ subcarriers separated by $15 \mathrm{kHz}$. The ideal carrier and sampling frequencies are $f_{c}=2.6 \mathrm{GHz}$ and $\frac{1}{T}=30.72 \mathrm{MHz}$, respectively. The length of the cyclic prefix is 144 samples and corresponds to approximately $7 \%$ of the OFDM symbol duration. A ring of $N_{T}=7$ base stations with an inter-site distance of $500 \mathrm{~m}$ transmits jointly to $N_{R}=7$ terminals using omni-directional antennas.

The radio channel is modeled by considering frequencyflat path loss with exponent 3.2 and shadow fading with $8 \mathrm{~dB}$ standard deviation and independent realizations between mobiles and base stations. Small-scale fading is modeled with impulse responses of independent circularly symmetric Gaussian distributed multipath components with an exponential power delay profile whose decay rate is $\frac{1}{2 \tau_{\mathrm{RMS}}}$, where $\tau_{\mathrm{RMS}}$ is the channel RMS delay spread. Independent realizations of the above model with 7 users positioned randomly within the 7cell environment were generated for building each instance of

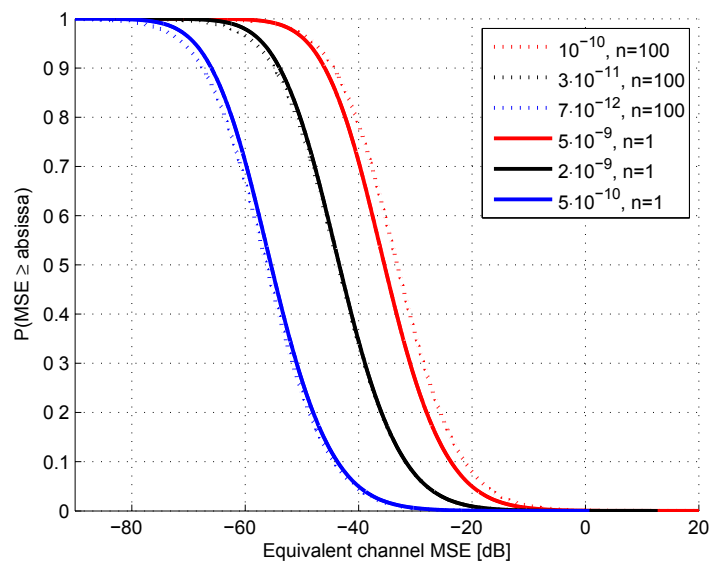

Fig. 1. Complementary CDF of the equivalent channel MSE due to base station-side CFO for the $1^{\text {st }}$ and $100^{t h}$ OFDM symbols (mobile terminals synchronized perfectly).

the channel matrix. Perfect channel knowledge was assumed at the base stations. Out-of-cluster interference is ignored.

The equivalent channel MSE is computed as $1 / S I R$, according to Eq. (10). It is computed per subcarrier for various cases of CFO and SFO. These impairments are assigned randomly and individually to base stations and mobiles, with normal distribution, zero mean and various (given) standard deviations. CFOs are specified directly as an RMS accuracy of the oscillator (for instance, a CFO RMS accuracy of $10^{-11}$ corresponds to $0.026 \mathrm{~Hz}$ for a carrier at $2.6 \mathrm{GHz}$ ). The contribution of SFO to the SIR is in general much smaller than the one due to CFO. Since both are locked to the same local reference oscillator, the sequel only reports on CFO results.

We assume that the base station oscillators are free running with respect to the distributed MIMO system. Their carrier frequencies and sample times may or may not be stabilized by a GPS signal or any other sort of beacon, but their variation over time is assumed to be slow enough to be considered static with respect to the OFDM signaling timescale. With free running base station-side oscillators the signals transmitted drift away in phase from each other as the OFDM symbol index $n$ grows. This drift is reset to zero when the precoder weights are updated. In LTE this is happening at best approximately every $7 \mathrm{~ms}$, which corresponds to roughly 100 OFDM symbols. We find that in order to serve $90 \%$ of the subcarriers for a duration of up to 100 OFDM symbols with a SIR due to CFO of at least $30 \mathrm{~dB}$ (equivalent to a channel MSE of $-30 \mathrm{~dB}$ ), the base station oscillators must have an RMS accuracy of $3 \cdot 10^{-11}$ or higher, as shown in Fig. 1. This level of accuracy can only be provided by rubidium oscillators or high-quality oven-controlled crystal oscillators (OCXOs) stabilized by GPS signals.

\section{Clustering of Base Stations and Interference ANALYSIS}

For the use of JT CoMP in practice the overhead for pilots, feedback and back-haul has to be kept within reasonable limits. 
The most efficient way to reduce overhead is to limit the cooperation area. Therefore base stations (BSs) are grouped into subsets denoted as clusters with joint processing allowed only between BSs belonging to the same cluster. BSs outside the cluster are not coordinated and thus cause residual interference. Furthermore, dynamic clustering allows a more efficient power allocation. Mobile users experiencing a weak channel to a given BS are assigned to another base stations in another cluster.

Real-world includes channel and synchronization impairments, where the clustering helps as well. Keeping the number of cooperating BSs low, limits the number of impairment sources and thus the performance degradation as well. For instance, in the case of synchronization errors, the dimension of the phase drift matrix $\Theta(k)$ grows with the number of BSs contributing to the CoMP transmission.

Ultimately, the cluster size defines the specifications needed for channel and synchronization impairments. When clusters are smaller, the margin relative to the cluster area is larger, i.e. out-of-cluster interference has more impact. Accordingly, the residual interference due to the impairments in an isolated cluster must not be larger than the out-of-cluster interference. Hence, the larger the cluster, the higher are the requirements on channel knowledge and synchronization impairments. including a iid Gaussian error as channel mismatch.

As a system model, we consider a cellular OFDM downlink where a central site is surrounded by multiple tiers of sites. We assume each site to be partitioned into three $120^{\circ}$ sectors, i.e. a set $\mathcal{L}$ consisting of $L=|\mathcal{L}|$ sectors in total. Each sector constitutes a cell, and frequency resources are fully reused in all $L$ cells. $\mathcal{K}$ represents the set of cells included in a given cluster and $K=|\mathcal{K}|$ denotes its maximum dimension. We assume disjoint clusters, i.e. a given BS cannot belong to more than one cluster operated at the same time/frequency resource. For OFDM systems, the overlap of multiple clusters can be achieved conveniently in the frequency domain.

In the $i$ th cluster, there are $K \mathrm{BSs}$, each one equipped with $N_{T}$ transmit antennas, while the $M$ users are equipped each with $N_{R}$ receive antennas. The users inside the cluster are served by signals jointly emitted from $K N_{T}$ transmit antennas, where $K \cdot N_{T} \geq M \cdot N_{R}$. The $K N_{T} \times K N_{T}$ precoding matrix $\mathbf{P}_{i}=\left[\mathbf{b}_{i, 1} \cdots \mathbf{b}_{i, M}\right]$ contains the precoders $\mathbf{b}_{i, m}$ designed for each of the users.

For further analysis, we assume the $i$-th cluster is surrounded by $L-K$ BSs evoking non-coordinated cochannel interference (CCI). Thus, the received downlink signal $\mathbf{y}_{m}$ at user $m$ in the cellular environment is given by

$$
\begin{gathered}
\mathbf{y}_{m}=\underbrace{\mathbf{H}_{i, m} \mathbf{b}_{i, m}}_{\overline{\mathbf{h}}_{m}} \sqrt{p_{i, m}} x_{i, m}+\underbrace{\sum_{\substack{j=1 \\
j \neq m}}^{M} \mathbf{H}_{i, m} \mathbf{b}_{i, j} \sqrt{p_{i, j}} x_{i, j}}_{\zeta_{m}} \\
+\underbrace{\sum_{\substack{\forall \in \mathcal{L} \backslash \mathcal{K} \\
l \in \mathcal{K}}} \sum_{j=1}^{N_{T}} \mathbf{H}_{l, m} \mathbf{b}_{l, j} \sqrt{p_{l, j}} x_{l, j}+\mathbf{n}}_{\mathbf{z}_{m}}
\end{gathered}
$$

The desired data stream $x_{i, m}$ transmitted to the $m$ th user from the $i$ th cluster is distorted by the intra-cluster and inter-cluster interference plus noise aggregated in $\zeta_{m}$ and $\mathbf{z}_{m}$, respectively. $\mathbf{H}_{i, m}$ spans the $N_{R} \times K N_{T}$ channel matrix for user $m$ formed by the $i$ th cluster and $p_{i, m}$ is its power allocation. Thus, $\zeta_{m}$ denotes the interference generated within the cluster.

As the number of spatial channels formed using linear beamforming is limited by the number of transmit antennas, the BSs select a set of active users for receiving data. This user selection could be done optimally using a brute-force search over all possible combinations of users. But due to the high complexity when the number of users is large, suboptimum techniques based on a greedy algorithm have been shown to provide near-optimum performance [13]. Extensions of the $\mathrm{ZF}$ technique to the case of multiple receive antennas appear in [14], [15]. Multiple spatial streams (or eigenmodes) are transmitted to each user without inter-user interference, resulting in a block diagonal (BD) covariance matrix. An extension of the BD concept, called multi-user eigenmode transmission (MET), uses ZF for maximizing the weighted sum rate. On a frame-by-frame basis, MET distributes up to $K N_{T}$ spatially multiplexed streams for one or multiple users. MET was initially proposed for multi-user MIMO (MU-MIMO) transmissions and its extension to JT CoMP case is summarized as follows. Assume that each user multiplies its channel matrix by the Hermitian of the left dominant eigenvector. The effective channel after the linear antenna combining can be written as

$$
\mathbf{h}_{k M E T}=\left(\mathbf{u}_{k}\right)^{H} \mathbf{H}_{k}=\left(\mathbf{u}_{k}\right)^{H} \mathbf{U}_{k} \boldsymbol{\Sigma}_{k}\left(\mathbf{V}_{k}\right)^{H}=\lambda_{k}\left(\mathbf{v}_{k}\right)^{H} .
$$

We use a distributed scheme, where $K$ BSs perform CoMP joint processing (JP) to a set $M$ active users each equipped with $N_{R}=2$ receive antennas. The scheme is evaluated with a system simulator using $L=57$ multi-antenna BS sectors and a wraparound technique using the 3GPP spatial channel model extended (SCME) channel model. We determine the system performance by assuming a dynamic and user-driven clustering method. The active set of users is selected according to the following metric: A set $\mathcal{M}=\mathcal{M}_{\text {all }}$ of active multiantenna terminals is uniformly distributed in the $i$-th cluster of the cellular environment. The user selection for each cell is done by a round-robin scheduling policy, yielding a set of users $\mathcal{M}_{k}$ of size $\left|\mathcal{M}_{k}\right|=N_{T}$. Note, the users in $\mathcal{M}_{k}$ experience highest channel gain to the $k$-th BS, i.e. all users are connected 


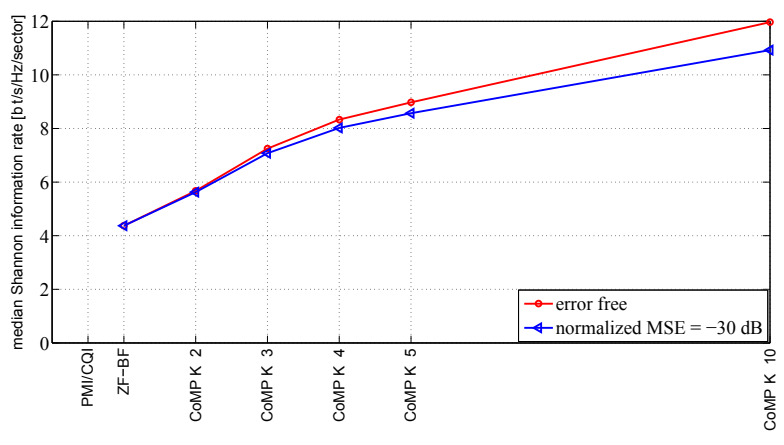

Fig. 2. Performance results as a function of the cluster size $K$. The normalized MSE is per subchannel, i.e. in case of $K=10$ sectors in the cluster, each equipped with $N_{T}=2$ antennas, the UE estimates up to $K \times N_{T}=20$ subchannels with an iid Gaussian normalized MSE.

to a master BS. In addition, all user sets $\mathcal{M}_{k}$ are disjoint for different BSs $k \in K$. Further, we emulate a cluster selection where the $K$ strongest channel gains of the users in $\mathcal{M}_{k}$ are the ones of the $K$ BSs within the cluster. Results are provided for different cluster sizes of $K \in\{1,2,3,4,5,10\}$. All results in Fig. 2 are based on an equal power per beam constraint with a per antenna power constraint (PAPC). Finally, we increase the cluster size from $K=1$ to $K=10$. Fig. 2 depicts the achievable Shannon information rate per sector as a function of the cluster size $K$ and accuracy of CSI feedback, i.e. in case of error free and erroneous CSI.

For erroneous CSI, we consider a Gaussian i.i.d. normalized MSE per sub channel, which results in a more severe degradation with increasing cluster size. From the Fig. 2 it is obvious that an MSE of $\mu=-30 \mathrm{~dB}$ is not a limiting factor for the system performance of JT CoMP at practical cluster sizes.

\section{COMPEnSATION}

Our trials in [6] have shown in principle that these impairment effects can be brought under control. However, we have used an idealized setup: GPS disciplined rubidium clocks, low phase-noise local oscillators, non-quantized feedback at pilot carrier sufficient for precise channel interpolation and static channel conditions. There is one unpredictable impairment, namely phase noise. Fortunately the larger group of impairments is predictable and can be compensated using advanced channel prediction and compression algorithms. This group covers frequency and timing errors, time variation of the channel and errors due to quantization. Developing efficient compensation algorithms is obviously a challenging task for future research.

\section{CONCLUSION}

We have studied the performance degradation due to impairments while using coordinated multi-point joint transmission in a multi-cell network. Impairments include channel estimation, feedback delay and synchronization errors among the base stations. We derived a generic model for the resulting interference between the data streams of multiple users served jointly by multiple base stations in the cluster. The resulting interference must not be larger than the one obtained after cooperation due to interference of non-coordinated cells surrounding the cluster. We have introduced an equivalent mean square error approach which can be used as a generic evaluation framework to evaluate the performance degradation due to various impairments.

\section{ACKNOWLEDGEMENTS}

The authors are grateful to the German Ministry of Education and Research (BMBF) for financial support through the national research project EASY-C as well as to the German Academic Exchange Service (DAAD) for financially supporting the cooperation between the Fraunhofer Heinrich Hertz Institute and the Pontificia Universitad Católica de Chile.

\section{REFERENCES}

[1] M. Costa, "Writing on dirty paper," IEEE Trans. Inform. Theory, vol. 29, no. 3, pp. 439-441, 1983.

[2] M. Sharif and B. Hassibi, "On the capacity of mimo broadcast channels with partial side information," Information Theory, IEEE Transactions on, vol. 51, no. 2, pp. $506-522,2005$.

[3] Q. Spencer, C. Peel, A. Swindlehurst, and M. Haardt, "An introduction to the multi-user mimo downlink," Communications Magazine, IEEE, vol. 42 , no. 10 , pp. $60-67$, oct. 2004.

[4] G. Foschini, K. Karakayali, and R. Valenzuela, "Coordinating multiple antenna cellular networks to achieve enormous spectral efficiency," vol. 153 , no. 4 , august 2006 , pp. $548-555$.

[5] V. Jungnickel, T. Wirth, M. Schellmann, T. Haustein, and W. Zirwas, "Synchronization of cooperative base stations," IEEE International Symposium on Wireless Communication Systems (ISWCS), Oct. 2008.

[6] V. Jungnickel, A. Forck, S. Jaeckel, F. Bauermeister, S. Schiffermueller, S. Schubert, S. Wahls, L. Thiele, T. Haustein, W. Kreher, J. Mueller, H. Droste, and G. Kadel, "Field Trials using Coordinated Multi-Point Transmission in the Downlink," in 3rd International Workshop on Wireless Distributed Networks (WDN), held in conjunction with IEEE PIMRC 2010. IEEE, Sep. 2010, pdf available.

[7] B. Zarikoff and J. Cavers, "Carrier frequency offset in coordinated multibase mimo narrowband systems," in Vehicular Technology Conference, 2008. VTC Spring 2008. IEEE, May 2008, pp. 872 -877.

[8] T. Koivisto and V. Koivunen, "Impact of time and frequency ofsets on cooperative multi-user mimo-ofdm systems," September 2009.

[9] C. Oberli, "ML-based tracking algorithms for mimo-ofdm," Wireless Communications, IEEE Transactions on, vol. 6, no. 7, pp. $2630-2639$, july 2007.

[10] K. Manolakis, C. Oberli, and V. Jungnickel, "Synchronization requirements for ofdm-based cellular networks with coordinated base stations: Preliminary results," 15th International OFDM Workshop, Hamburg, Aug. 2010.

[11] V. Pohl, P. Nguyen, V. Jungnickel, and C. von Helmolt, "Limits of the Achievable Symbol Rate in Flat Fading MIMO Systems," in Proc. 14th IEEE Intern. Symposium on Personal, Indoor and Mobile Radio Commun. (PIMRC), vol. 3, Bejing, China, Sep. 2003, pp. 2548-2552.

[12] 3GPP LTE, E-UTRA and E-UTRAN - Overall description (Release 10), Std., Jun. 2010.

[13] F. Boccardi, F. Tosato and G. Caire, "Precoding Schemes for the MIMOGBC," IEEE International Zurich Seminar on Communications, Feb. 2006.

[14] H. Viswanathan, S. Venkatesan and H. Huang, "Downlink Capacity Evaluation of Cellular Networks With Known-Interference Cancellation," IEEE J. Sel. Areas Comm., vol. 21, no. 5, pp. 802-811, June 2003.

[15] Q. H. Spencer, A. L. Swindlehurst and M. Haardt, "Zero-Forcing methods for Downlink Spatial Multiplexing in Multiuser MIMO Channels," IEEE Trans. on Signal Processing, vol. 52, no. 2, pp. 461-471, Feb. 2004. 